Rapport - Société canadienne d'histoire de l'Église catholique

\title{
Monseigneur Dominique Racine, fondateur de l'Église Saguenéenne
}

\section{Jean-Claude Drolet}

Volume 31, 1964

URI : https://id.erudit.org/iderudit/1007346ar

DOI : https://doi.org/10.7202/1007346ar

Aller au sommaire du numéro

Éditeur(s)

La Société canadienne d'histoire de l'Église catholique

ISSN

0318-6148 (imprimé)

1927-7075 (numérique)

Découvrir la revue

Citer cet article

Drolet, J.-C. (1964). Monseigneur Dominique Racine, fondateur de l'Église

Saguenéenne. Rapport - Société canadienne d'histoire de l'Église catholique, 31, 55-64. https://doi.org/10.7202/1007346ar

Tous droits réservés @ La Société canadienne d'histoire de l'Église catholique, 1965
Ce document est protégé par la loi sur le droit d'auteur. L'utilisation des services d'Érudit (y compris la reproduction) est assujettie à sa politique d'utilisation que vous pouvez consulter en ligne.

https://apropos.erudit.org/fr/usagers/politique-dutilisation/ 


\title{
Monseigneur Dominique Racine fondateur de l'Église Saguenéenne
}

\author{
Cet exposé sur $M^{g r}$ Dominique Racine, fondateur de l'Eglise \\ saguenéenne, présente les grandes lignes d'une thèse pour \\ lobtention d'un Doctorat ès Lettres histoire à l'Université Laval. \\ La Société historique du Saguenay et le Séminaire de Chicou- \\ timi ont fourni la plus grande partie des documents nécessaires \\ à ce travail.
}

C'est avec un très vif et croissant intérêt, avec une grande édification que nous offrons à tous ceux qui s'intéressent à l'histoire religieuse de notre pays, cette brève étude sur $M^{\mathrm{gr}}$ Dominique Racine, qui a fait une grande et belle œuvre et qui a été désigné, à juste titre par l'auteur de sa notice biographique publiée après sa mort, "l'apôtre du Saguenay ".

Cet évêque a été un facteur puissant dans l'établissement et le développement de cette vaste région appelée "Royaume du Saguenay 》. Mais $\mathrm{M}^{\mathrm{gr}}$ Racine : qui est-ce exactement ? Le Séminaire de Chicoutimi conserve précieusement son cœur dans sa magnifique chapelle, la rue commerciale et une imposante école secondaire perpétuent son nom. C'est pour combler une lacune évidente dans l'historiographie saguenéenne et de l'Eglise canadienne que nous présentons cette modeste conférence, synthèse d'un travail plus élaboré.

Situons rapidement dans l'espace et dans le temps, le personnage dont nous voulons esquisser les traits principaux et l'œuvre immense.

En réalité, le Saguenay, qui signifie en langue algonquine "d'où l'eau sort ", est d'abord une vaste région, une région immense à laquelle la légende et l'histoire ont donné le titre de « Royaume ».

Dans ses dimensions, par son étendue et ses ressources, par sa situation géographique et ses notes caractéristiques, c'est tout un pays. Un de ses traits marquants est la variété. Le Saguenay présente une sorte de triptyque : trois régions de caractère différent : la Côte Nord, ouvrant sur le large horizon marin; le haut-Saguenay, plateau de soleil et d'espace où s'enchâsse comme une perle dans un écrin, le lac SaintJean; le bas-Saguenay, ou secteur de Chicoutimi qui sépare et unit les deux autres. Pays où alternent et se complètent les montagnes et les plaines, la mer et le plateau, l'activité et la solitude, le cycle à la fois capricieux et stable des saisons. Pays pourvu en plus d'un fjord merveilleux, seule route de l'époque, et doté d'un ciel aux riches couleurs et aux mille aspects qui est comme un cinéma perpétuel. Une autre de ses notes particulières, c'est qu'il est à la fois un pays neuf et un pays très vieux. 
Au point de vue géologique, il fait partie du bouclier canadien, qui est le plus vieux sol du monde; ses montagnes de granit précambrien, toutes arrondies et usées par le temps, appartiennent aux premières assises solides de la terre. Par contre, une section, dont Chicoutimi, occupe l'entrée et le lac Saint-Jean, le centre, présente les formations géologiques les plus récentes. De même au point de vue historique, l'histoire du Saguenay commence avec celle de la Nouvelle-France et même avant. Mais ce pays est resté pendant plus de trois siècles à l'état sauvage, en marge du reste de la Nouvelle-France; comme pays colonisé, il est donc tout jeune. Son territoire isolé par les conditions géographiques et par la diplomatie indienne qui empêchait les Blancs d'y pénétrer, était un pays fermé, réservé même comme domaine personnel du Roi à partir de 1674, pour la traite des pelleteries. On n'y tolérait que le personnel des postes de traite pour le commerce, et les missionnaires pour l'évangélisation des Indiens. D'ailleurs,

- à plus d'un point de vue, la colonisation du Saguenay a reproduit fidèlement, sur un théâtre moindre, sans doute, les circonstances de la fondation de la Nouvelle-France ${ }^{1}$,.

Sur ce vaste théâtre, l'abbé Dominique Racine et plus tard $M^{\text {gr }}$ Racine allait déployer à son aise le zèle, l'énergie, l'activité infatigable et l'esprit d'initiative dont il était capable.

Les premières années de sa vie ressemblent à celles de plusieurs. Il est né à Saint-Ambroise de la Jeune Lorette, le 24 janvier 1828, de Michel Racine, modeste forgeron, et de Louise Pépin. Orphelin de père alors qu'il est encore très jeune, il sera l'objet de soins particuliers de la part de sa mère et de ses frères aînés. Son premier instituteur est un M. Lefrançois, son parrain, un homme très digne. A dix ans, soit en 1838, il fait sa première communion, et est enfant de chœur à la chapelle des Hurons de la Jeune Lorette. 1838 est une année qui mérite d'être soulignée pour deux raisons : son frère aîné Michel reçoit l'ordination sacerdotale et les premiers colonisateurs entament la forêt sauvage du Saguenay, préparant ainsi le vaste champ d'action du futur curé et évêque.

A douze ans, le Séminaire de Québec lui ouvre ses portes et lui permet de poursuivre ses études classiques. Des prêtres de talent et de dévouement le forment ainsi pendant huit ans. Toute sa vie, il gardera une affection profonde et sincère pour son Alma Mater. Ecrivant, le 26 avril 1875, à l'abbé Cyrille Légaré au Séminaire, il lui dit :

....j'éprouve un plaisir indicible à me rappeler les heureux jours que j'ai passés au Séminaire. Et je remarque que plus les années s'écoulent, plus j'affectionne ces années trop vite écoulées, plus je me sens attaché à votre maison bénie et à tous ceux avec qui j'aurai été en relation. La pensée du Séminaire de Québec, de nos regrettés Supérieurs et Directeurs, des anciens amis, voilà la source de mes consolations au milieu de mes peines et de mes misères 2 ,

1 V.-A. Huart, Notes biographiques de $M^{\text {or }} D$. Racine, p. 32.

2 ASQ Lettres, Carton N, n⿳ 143, 26 avril 1875. Monseigneur D. Racine à l'abbé Cyrille Légaré. 
Deux autres faits de sa vie d'étudiant au Séminaire doivent être soulignés : au mois de septembre 1844, il assiste à l'ordination sacerdotale d'un deuxième frère, Antoine, qui le devancera au pays du Saguenay et aussi dans l'épiscopat; quelques mois plus tard, la mort emporte, en pleine activité, l'abbé Michel, à peine âgé de 30 ans. Ces deux événements ont, certes, une influence sur l'âme du jeune Dominique.

On note aussi que pendant la dernière année de ses études, soit de 1848 à 1849, il est rédacteur de la publication collégiale «l'Abeille »; il est préfet de la Congrégation et aussi désigné comme président de la société typographique ${ }^{3}$. Dominique Racine a donc fait des études sérieuses interrompues par quelques maladies. Il est certes intéressant de noter ici l'atmosphère particulière de la classe des finissants de Dominique Racine; car sur huit confrères, sept répondent à l'appel au sacerdoce. L'un d'eux est Thomas-Etienne Hamel, futur Recteur de l'Université Laval; se noue entre ces deux confrères une amitié qui ne cessera qu'à la mort de l'évêque; la correspondance nous dévoile une tendre affection et une chaleur de sentiment vraiment remarquables. Au Séminaire, le jeune Dominique jouit d'une affection toute paternelle et d'une sollicitude particulière de M. l'abbé L.-J. Casault, et de M. Holmes.

Septembre 1849 marque son entrée au Grand Séminaire de Québec. Pendant ses études théologiques, il remplit des fonctions variées : d'abord de 1849 à 1852 , il est surveillant ou * maître de salle » avec zèle et dévouement; en 1853, on le retrouve à l'Archevêché de Québec en qualité de secrétaire. Il est ordonné prêtre le 24, septembre 1853. Les études théologiques du jeune lévite sont certes tronquées et insuffisantes, il est quelque peu autodidacte, mais c'est une méthode de formation à la mode, à l'époque, méthode qui a des avantages mais aussi des inconvénients.

Les premières années de son sacerdoce ressemblent à celles de la plupart des prêtres du ministère, excepté qu'elles sont marquées déjà par des réalisations qui indiquent l'homme du coup d'œil et de l'action.

Pendant les cinq premières années, il est vicaire à la paroisse de Notre-Dame de Québec dirigée par le curé J. Auclair. Une sainte amitié ne tarde pas à se nouer entre ces deux hommes d'élite.

- Le curé Auclair, dit un vieillard, adorait son jeune vicaire; et il se plaisait beaucoup à l'entendre prêcher et le félicitait. Une fois que l'abbé Racine avait fait un beau sermon, le curé Auclair lui donna une montre en or, en lui disant: Vous l'avez bien gagnée ${ }^{4}$.

Les deux œuvres qui absorbent surtout le temps et les forces de l'abbé Racine sont la confrérie de la Sainte-Famille et la restauration du pieux et antique sanctuaire de Notre-Dame des Victoires à la Basse-Ville. Il semble avoir eu un brillant ministère dans cette paroisse en vue de la ville de Québec, il a observé, travaillé, il est alors prêt pour diriger une

3 L'Abeille, vol. $1, \mathrm{n}^{\circ \mathrm{O}} 7,14$.

4 SHS Mémoire 169, par. 95. 
cure. En 1858, soit à l'âge de trente ans, on le désigne comme curé de Saint-Basile de Portneuf. C'est avec peine qu'il quitte son premier vicariat et s'éloigne de confrères qui lui sont très chers. Là, il construit le presbytère avec ses propres ressources. Puis de 1859 à 1862 , il est curé de Saint-Patrice de Rivière-du-Loup ou Fraserville. Il achève la construction de l'église, fonde en 1860 le couvent confié aux Religieuses du Bon-Pasteur et s'y intéresse activement. Des liens profonds et durables unissent le curé et les paroissiens, il est un pasteur dévoué, zélé, actif, affectueux, les paroissiens lui sont soumis avec respect.

Enfin le curé Racine est nommé à Chicoutimi en 1862. Le reste de sa carrière, soit vingt-six ans, appartient désormais à la paroisse et au diocèse de Chicoutimi.

En 1862, Chicoutimi a vingt ans d'existence. C'est une paroisse qui sort à peine de la période de colonisation. Son village ressemble à un " rang " aux maisons espacées le long d'un chemin de terre, sans trottoirs, avec des granges et des hangars ici et là, une église vers le centre et des agglomérations aux deux extrémités, soit Rivière-du-Moulin et le Bassin, dans le voisinage des scieries. Cette cure semble causer bien des soucis au jeune curé. Le 5 décembre 1865, il écrira à l'abbé B. Paquet, alors à Rome :

- Oui, je suis encore à Chicoutimi; et Dieu sait combien de temps j'y serai encore. Comme il ne me consulte pas, je me soumets volontiers; mais, si l'on me demandait une fois mon avis, je me déchargerais promptement de cette cure. Que tu es heureux de n'avoir pas la responsabilité d'une grande paroisse sur les épaules 5 ! »

La première réalisation importante du curé Racine est d'établir un pensionnat pour les jeunes filles. En 1864, le couvent du Bon-Pasteur ouvre ses portes.

Un événement tragique est l'occasion de faire valoir la puissance de son action naturelle et surnaturelle : le feu du 19 mai 1870, qui balaie toute la partie colonisée du Saguenay, de Mistassini à la Baie des $\mathrm{Ha}, \mathrm{Ha}$, environ 1,500 milles de superficie. Vers la fin de la journée, le feu approche de Chicoutimi. Monsieur William Price, un protestant, accourt au presbytère pour demander au curé sa protection. Les témoins du fait attribuent à son intervention directe, la préservation du village de Chicoutimi et les rapports de l'époque nous apprennent qu'il est l'âme des secours qui sauveront les populations en détresse.

Puis sa charge de Vicaire forain et de Vicaire général à partir du 19 mars 1871 - cette dernière fonction est une preuve non équivoque de l'estime et de la confiance de l'Archevêque de Québec - l'appelle à participer à toutes les activités de la région en pleine croissance. A ce double titre, il fait fidèlement la visite des paroisses et adresse à l'Archevêché de Québec des rapports où sont nettement indiqués l'état et les besoins de chacune. Il a l'honneur de présider à la naissance de plusieurs belles paroisses de la région.

5 ASQ Université 104, 85. 
En 1873, avec la permission et le concours de son Archevêque, il installe dans une maison d'école du village de Chicoutimi, le Séminaire; il y consacre ses ressources, son intelligence, son cœur, ses mains, car l'année suivante, il commence une construction en pierre. Depuis 1865, il rumine ce projet et en fait part à son ami l'abbé T.-B. Hamel, du Séminaire de Québec :

- Je suis poursuivi par cette idée, lui écrit-il le 4 mars 1866 , et plus j'y pense plus je trouve que s'il est une partie du Diocèse qui ait besoin d'un collège, c'est le Saguenay. Que je voudrais être riche, comme il s'en trouve sous la calotte du ciel, j'aurais bientôt mis hache en bois. Malheureusement ma bourse n'est pas aussi grande que mes désirs; et j'ai encore des créanciers qui crient après moi. Malgré cela, je suis bien prêt à faire des sacrifices pour la réalisation de ce projet ${ }^{6}$.,

Il résidera dans son cher Séminaire jusqu'en 1887; premier Supérieur, il rédige les Constitutions, établit et fixe l'esprit et la tradition de la maison et se plie lui-même aux exigences de la vie en communauté. Sur son lit de mort, il s'écrie :

- Mon séminaire... Mon séminaire, comme je l'aimais! Je puis dire de lui, comme Adam de la compagne que Dieu lui avait donnée : C'est l'os de mes os, la chair de ma chair ! J'aurais voulu faire pour lui beaucoup plus que je n'ai fait. Dieu ne l'a pas permis ?.,

Ce sont ces paroles, d'ailleurs, qui inspireront aux prêtres du Séminaire de demander à son frère $\mathrm{M}^{\mathrm{gr}}$ Antoine, évêque de Sherbrooke, de laisser le cour de l'illustre défunt au Séminaire de Chicoutimi.

De 1876 à 1878 le curé Racine dirige la construction d'une nouvelle église paroissiale; à son insu ce sera la cathédrale d'un nouveau diocèse.

Depuis 1871, il est question de former un évêché dans le Saguenay. M. le Grand Vicaire Racine s'élève contre ce projet, car c'est selon lui, une source de ruines. Le 24 mai 1878 , Rome a décidé de former ce diocèse et le curé Dominique Racine en sera le premier évêque.

- A vrai dire, personne ne fut surpris de cette nomination; tout le monde en était certain à l'avance. Ses qualités et ses vertus éminentes, les grandes œuvres qu'il avait déjà accomplies pour l'avantage matériel du Saguenay le désignaient manifestement pour occuper cette position supérieure ${ }^{8}$.,

Cependant l'évêque élu pense bien autrement, dans son mandement d'entrée :

-... Toutes nos objections et nos résistances étant restées sans effet, nous avons dû nous soumettre et dire encore avec Jésus-Christ : Mon Dieu, que votre volonté soit faite et non pas la mienne.»

-... Rien, en effet, dans les nombreuses occupations auxquelles nous avons dû nous livrer dans les divers postes qui nous ont été confiés jusqu'à présent, ne pouvait nous faire prévoir que nous fussions appelés à devenir votre premier pasteur ${ }^{9}$.

6 ASQ Univ. 80, 36, 4 mars 1866.

7 L'Oiseau-Mouche, 29 sept. 1900. Vol. VIII, $\mathrm{n}^{\circ} 14$.

8 V.A. HuART, op. cit., p. 47.

- ABC Registre d'insinuation, 10-11. 
Il connaît assez bien la lourde responsabilité qui pèsera désormais sur ses épaules, ce qui explique ses paroles à $\mathrm{M}^{\text {sr }}$ Fabre, Archevêque de Montréal :

- De ce temps-ci, des paroles de condoléances sont plus en harmonie avec les sentiments de mon âme que des félicitations. Sans avoir goûté au plat dont se nourrissent quotidiennement, nos Seigneurs les Evêques, je l'ai approché d'assez près pour ne pas ignorer qu'il renferme beaucoup d'amertume. Et c'est bien là une des causes de mon affliction 10.,

\begin{abstract}
- In francaic, Pierre I Lernv, arrivé an Canada denuis aueloues années. pour $\mathrm{y}$ introduire un nouveau système d'enseignement, s'est fait connaître par ses excentricités. Il a cru devoir s'opposer à la nomination de $\mathrm{M}^{\mathrm{gr}}$ Dominique Racine, comme évêque de Chicoutimi. Dans son opinion, on aurait dû élever sur ce siège épiscopal, le R.P. Arnaud, o.m.i., missionnaire à Betsiamis ${ }^{11}$. 》
\end{abstract}

Essayons d'esquisser maintenant le portrait moral de ce nouvel évêque. Au point de vue moral, ses traits caractéristiques sont la patience, la bonté, une charité sans limites, une complète abnégation et un perpétuel esprit de sacrifice et de mortification, car la pauvreté est la compagne de toute sa vie. Il ne manque ni de tact, ni de sensibilité, ni même d'affection et de tendresse. Mais il a un tempérament vif et impétueux. Son abord, dès son jeune âge, donne l'impression d'un homme énergique et décidé. Il déploie une persévérance énergique dans ses projets, son activité est débordante; activité incomparable, capable de porter son attention et ses soins jusque sur les moindres détails et de ne rien négliger dans l'administration d'une vaste paroisse et plus tard dans l'érection et l'administration plus complexe d'un jeune diocèse immense en étendue. Créer, bâtir, réglementer, réformer, écrire, semble une nécessité pour lui. Activité aussi entreprenante et hardie de celui qui a la rude tấche d'être, semble-t-il, le porte-parole de son Archevêque pendant une période difficile de l'histoire de l'Eglise québecoise, activité infatigable que seule la mort arrêtera. Enfin, homme de bonne humeur, il aime taquiner, s'amuser avec ses confrères et ses amis, et même ses paroissiens et diocésains. D'ailleurs son prestige est vraiment extraordinaire; il a la vénération et l'affection de ses diocésains; son portrait en grand, bien encadré, est à la place d'honneur dans toutes les maisons.

Au point de vue intellectuel, ses qualités essentielles sont l'ordre, la logique, la précision. La formation théologique et littéraire lui a certes fait défaut, il s'en plaint lui-même. Il possède une a promptitude d'esprit » lui permettant de démêler les situations les plus compliquées et une rectitude de jugement remarquable. A cela, il faut ajouter un talent oratoire hors pair, car il possède à un haut degré les qualités extérieures d'un orateur,

- un maintien rempli de dignité, un regard qui pouvait aussi bien porter

l'émotion dans les cœurs et la terreur dans les esprits, une voix au timbre

10 AAM Dossier 1878, Lettre de Mor D. Racine à Mor Fabre, datée du 2 juillet 1878.

11 ASC Ann., Vol. 1, p. 229. 
magnifique, vibrant dans le pathétique, lançant la foudre dans les consciences coupables, et le geste venant avec toutes ses ressources doubler l'effet de la parole. Sa phrase, limpide et vive, imagée et sachant à l'occasion se parer des magnificences du style, servait un aliment toujours approprié aux intelligences qui recueillaient avidement ces enseignements de salut... Son talent d'improvisation était surtout remarquable 12 ,.

Au physique, on le décrit sur son premier passeport comme un homme de cinq pieds onze pouces, aux yeux bleus, à la tête blanche, de stature robuste et d'une très belle apparence ${ }^{13}$. On lit dans un journal de l'époque

- qu'on était à demi vaincu de suite par le charme de cette physionomie, et tous, les humbles comme les grands, et, pourquoi ne le dirions-nous pas, depuis l'ecclésiastique de son diocèse jusqu'à Sa Sainteté Léon XIII, tous ont su apprécier la franchise de son commerce ${ }^{14}$.

Grand par ses qualités intellectuelles et morales, $\mathrm{M}^{\mathrm{gr}}$ Dominique Racine l'est aussi par ses œuvres. Le premier évêque d'un nouveau diocèse a beaucoup à créer et à organiser; en ce domaine, les dix années de l'épiscopat du nouvel élu sont fort bien remplies.

Son diocèse est très vaste, comprenant toute l'étendue du \& Royaume du Saguenay », avec en plus le comté de Charlevoix. Ce diocèse compte alors vingt-sept paroisses; $M^{\text {gr }}$ Racine en organisera onze nouvelles. Il les parcourra toutes trois fois en visite pastorale; les circonstances, les nécessités, le ramèneront plus d'une fois dans plusieurs d'entre elles. Il ne faut pas oublier surtout que ces courses dans le plus étendu des diocèses de l'époque, se font en voiture à cheval, par des chemins d'été ou d'hiver, dont la réputation est mauvaise et bien méritée.

Deux voyages à Rome en 1883 et 1885 pour les besoins du clergé et du diocèse et les intérêts religieux de la province, et plus d'un voyage chaque année à Québec, s'ajoutent au programme de ses randonnées.

Arrêtons-nous quelques instants sur l'œuvre de $\mathrm{M}^{\mathrm{gr}}$ Racine au sein de l'Episcopat québecois fortement divisé à cette époque. Les sources de division ne manquent pas d'ailleurs : il y a d'abord la Succursale de l'Université Laval à Montréal, le problème de l'influence indue, le libéralisme, l'érection du diocèse de Nicolet et bien d'autres questions secondaires se rattachant à ces principales. Nos recherches actuelles nous permettent de croire que $\mathbf{M}^{\mathrm{gr}}$ Dominique Racine est le porte-parole de l'Árchevêque de Québec auprès de Rome. Ces deux voyages l'attestent de même qu'une abondante correspondance. Il est plus difficile cependant de discerner les raisons du choix de l'évêque de Chicoutimi pourtant fort occupé par l'administration et le développement de son diocèse. D'ailleurs, il écrit lui-même à S.S. Léon XIII :

- Je n'ai, T.S. Père, aucun intérêt quelconque dans aucune des questions qui nous agitent; mon diocèse est trop séparé des autres, les voies

12 V.-A. Huart, op. cit., 91-92.

13 ASC Passeport du 5 septembre 1882

14 Justice, 30 janvier 1888, dans VS, t. III, 235-236. 
de communication qui nous relient aux grandes villes trop difficiles, la classe instruite trop livrée aux intérêts de leurs localités respectives pour que ces difficultés nous préoccupent d'une manière particulière. Si donc je suis revenu à Rome plaider la cause que je soutiens, c'est uniquement parce que je vois l'autorité du St-Siège méconnue, sa parole méprisée et le salut des âmes en péril 15. .

Nous croyons que son affection et son grand intérêt pour tout ce qui touche le Séminaire de Québec et l'Université Laval, son tempérament vif et impétueux, ferme et décidé, l'amitié profonde qui unit le Métropolitain ct le suffrazañt, quićbccois par naaissance, toutcs ces raisons, à notre humble avis, motivent son rôle très important mais très délicat. De plus, $\mathrm{M}^{\mathrm{gr}}$ Racine expose tous ces problèmes complexes et sérieux avec clarté et force détails et se permet même à un moment de suggérer au Saint-Père des remèdes aux maux qui troublent évêques et fidèles. Il épouse la cause de Québec dans ce conflit...

- Pauvres gens de Montréal ! écrit-il en 1878, on les dirait tous frappés d'aveuglement, et pourtant ils se croient la lumière du monde et ils ont la grande humilité de le crier sur les toits ${ }^{16}$.,

Mais malgré tout, $\mathrm{M}^{\mathrm{gr}}$ Racine semble poursuivre un seul but : celui de la paix et de la concorde entre les Evêques du Québec. C'est encore lui qui, en son nom et avec toute la sincérité qu'on lui connaît, demande au cardinal Simeoni la pourpre cardinalice pour $\mathrm{M}^{\mathrm{gr}}$ Taschereau, car

- ses éminentes qualités, sa grande intelligence, sa science profonde, ses hautes vertus le recommandent certainement à l'attention de l'illustre Léon XIII et le rendent digne d'une telle faveur. De plus, ajoute-t-il, l'élévation de Sa Grandeur à l'éminente dignité du Cardinalat contribuerait pour beaucoup à ramener la paix au milieu de nous ${ }^{17}$.

Le 10 juin 1886, il écrira de nouveau au Pape pour le remercier de l'élévation de $\mathrm{M}^{\mathrm{gr}}$ Taschereau au cardinalat.

Dès le début de son épiscopat, $\mathrm{M}^{\mathrm{gr}}$ Racine complète l'œuvre du Séminaire par l'organisation d'un Grand Séminaire et il aura le bonheur de donner l'onction sacerdotale à trente-quatre nouveaux prêtres.

La liste de ses communications personnelles au clergé et aux fidèles de son diocèse comprend soixante-seize documents : vingt-deux mandements, sept lettres pastorales, quarante-sept circulaires. Sa correspondance personnelle contient un nombre imposant de lettres. Il organise et suit de près les retraites, les conférences ecclésiastiques, les examens et les sermons des jeunes prêtres, la bonne tenue des registres et des comptes paroissiaux, la bonne marche de son Séminaire et des différentes institutions ainsi que les problèmes de l'Episcopat de l'époque.

Nombre d'autres œuvres sollicitent son attention et ses directives : celle de la propagation de la foi, en 1878; l'association des Enfants de

15 ASQ Univ., 28, 94, p. 133.

16 ASQ Univ., 49, 87, lettre du 20 novembre 1878.

17 AEC Registre des lettres 1 , p. 265. 
Saint-Joseph, pour les jeunes gens, le 19 mars 1880; celle des Enfants de Marie, le 8 mai de la même année; l'association des messes, en 1881; l'érection de la statue de Notre-Dame du Saguenay au cap Trinité, bénite par lui-même le 15 septembre 1881 ; la société de colonisation, sous le patronage de la sainte Famille, fondée le 21 février 1882; la tempérance, le Tiers-Ordre et l'adoration réparatrice en 1883; la confrérie de la Sainte-Face de Notre-Seigneur, établie en 1886 et longtemps en honneur dans toutes les familles; enfin l'œuvre de Saint-FrançoisXavier pour édifier la cathédrale.

Membre du bureau d'Education du Saguenay, $M^{\text {gr }}$ Racine donne aux écoles et à l'éducation une attention toute vigilante et particulière. Au Séminaire, il établit la congrégation de la Sainte-Vierge, le 25 décembre 1880, met les études officiellement sous le patronage de saint Thomas d'Aquin, en 1881, fait ériger l'institution en corporation civile en 1879. C'est encore lui qui dote la région du pensionnat et de l'école ménagère des Ursulines à Roberval, en 1882. En 1884, il attire à Chicoutimi les religieuses de la Miséricorde pour prendre charge de l'hôpital de la Marine qui deviendra plus tard l'Hôtel-Dieu Saint-Vallier.

L'action de $\mathrm{M}^{\mathrm{gr}}$ Racine en dehors du domaine proprement religieux et éducationnel pour le bien de la région est considérable. Parmi les œuvres matérielles auxquelles il a consacré beaucoup d'efforts, mentionnons le développement de l'agriculture, l'amélioration du système de voirie, la construction d'une voie ferrée pour relier la région à la capitale. Il n'y a presque pas de réalisations importantes auxquelles il n'a pas pris une large part.

L'œuvre apostolique de $\mathbf{M}^{\mathrm{gr}}$ Dominique Racine n'est pas seulement étendue : elle est d'une portée immense. C'est l'œuvre du pionnier, du fondateur; œuvre de base qui n'est pas partout complète et ne pouvait l'être à ce moment, mais œuvre qui dépasse par sa portée la période de la carrière épiscopale de son auteur et lui mérite le titre de fondateur de l'Eglise saguenéenne. Il est un grand évêque parce que son action tenace, énergique, s'exerce pour un juste et noble but : service de Dieu et de l'Eglise; la paix et la concorde dans l'Eglise québecoise de son époque.

"Le grand évêque - dit l'auteur de sa notice biographique - a été la personnification vraie et vivante, l'expression la plus heureuse peut-être et la plus complète d'un clergé unique dans les annales de l'Eglise, le clergé canadien... Il a été à un rare degré, avant tout deux choses : l'homme de Dieu et l'homme du peuple... Sa double carrière éclate comme une carrière de sainteté et comme une carrière de patriotisme ${ }^{18} \ldots$. 
Un homme n'est vraiment grand que par le cœur, mais le cœur luimême n'est vraiment grand, large, à la mesure des besoins supérieurs de l'humanité que s'il est marqué à l'effigie divine, que s'il est mû par l'inspiration surnaturelle. Il est facile de reconnaître cette effigie et cette inspiration dans le cœur de $\mathrm{M}^{\mathrm{gr}}$ Dominique Racine, fondateur de l'Eglise saguenéenne.

Jean-Claude Drolet, professeur

Ecole normale Cardinal-Bégin Arvida, P.Q. 